\title{
Using the PCSWMM 2010 SRTC Tool to Design a Compost Biofilter for Highway Stormwater Runoff Treatment
}

\section{Karen Finney and Bahram Gharabaghi}

Highway stormwater runoff adversely affects the water quality of receiving lakes and rivers. Pollutants that build up on roads and other impervious surfaces are harmful to aquatic life and surrounding ecosystems (Makepeace, 1995; Wiland and Malina, 1976; Pitt, 2003). Woody compost or overs has been found to be effective in removing highway pollutants, including suspended solids, heavy metals and petroleum hydrocarbons. Overs has also been found to be effective as a filtration medium when assembled inside a mesh casing to form a compost biofilter. However, it is important that the biofilter is sized properly to avoid overtopping during large storm events. This chapter discusses the application of the new sensitivity based radio tuning calibration (SRTC) tool in PCSWMM 2010 for the design of a compost biofilter for highway stormwater runoff treatment.

\subsection{Introduction}

Stormwater runoff originating from highways and other urban impervious surfaces has been identified as a significant source of non-point pollutants found in streams and rivers throughout Canada and the United States. Pollutants found in the runoff include suspended solids, heavy metals and petroleum hydrocarbons.

Woody compost (overs) is effective in removing highway pollutants such as these (Finney et al., 2010). Overs is effective as a filtration medium when assembled inside a mesh casing to form a compost biofilter. Compost biofil-

Finney, K. and B. Gharabaghi. 2011. "Using the PCSWMM 2010 SRTC Tool to Design a Compost Biofilter for Highway Stormwater Runoff Treatment." Journal of Water Management Modeling R241-09. doi: 10.14796/JWMM.R241-09.

(C) CHI 2011 www.chijournal.org ISSN: 2292-6062 (Formerly in Cognitive Modeling of Urban Water Systems. ISBN: 978-0-9808853-4-7) 
ters consist of two main components: a mesh photodegradable fabric known as a sock, and organic woody compost known as overs (Taleban et al., 2009; Finney et al., 2010). Individual compost biofilters are assembled by blowing the overs into a sock to form a sausage-like filter. Socks are available in several sizes and can be stacked to create a larger filter for a specific site.

\subsection{Objectives}

This chapter will demonstrate the SRTC tool's ability to assist in the calibration of gauge and flow data collected from a sampling site. The site from which the data was obtained was a compost biofilter project.

To implement new low impact developments (LIDs), standards for installation must be developed to ensure the technology will be effective. In the case of the compost biofilter it is important that the biofilter dam does not overtop due to high flow rates. One method of determining the potential flow-through rates of a stormwater conveyance system in a particular area is to model the site and the site details using a hydraulic modeling program like PCSWMM.

The specific objectives for the highway remediation project were to determine the flow-through properties of the biofilter, and to develop a simple design method for site-specific applications.

\subsection{Background}

The full-scale biofilter site was located on Highway 8 in Kitchener, Ontario (detailed in Finney et al., 2010). The site had average traffic of approximately 100000 vehicles/day and the catchment area was 0.85 ha. The site was fully equipped with flow gauges, level loggers and weirs. A tipping bucket raingauge was also assembled close to the site. A large compost biofilter was installed in the stormwater drainage ditch beside the highway. Water samples were collected before and after the biofilter on the stormwater flow path. Samples were tested for total suspended solids, heavy metals (zinc and copper) and petroleum hydrocarbons, and were analyzed for particle size distribution. Two flow loggers and two v-notch weirs recorded the hydrograph time series for locations pre and post biofiter treatment. The compost biofilter was installed in July 2008. A Filtrexx blower truck was used to assemble the filter bags on site. 


\subsection{PCSWMM 2010}

When designing a compost biofilter for an application such as in a ditch on a highway it is important that the site's flow characteristics are determined in order to be able to design a biofilter that will not overtop. The most important information for designing a check dam biofilter is the maximum flow rate to which the biofilter is subjected. In this example, the Highway 8 remediation site described above is modeled, and the model is calibrated using observed inlet flow rates and surveying data. Once calibrated, a known design sized storm is introduced into the model together with the calibrated site parameters in order to obtain a peak flow rate for which the biofilter is to be designed. Although the compost biofilter was installed onsite prior to this exercise, the methodology of compost biofilter design can be demonstrated for this site using the compost biofilter's flow-through characteristics. The data required for this project include: soil characteristics, cross sectional transect data, percentage of directly connected impervious area as well as Manning's coefficients. This information was defined for the model by surveying the site and making assumptions based on the literature. A raingauge close to the site as well as onsite weirs and loggers provided the rainfall data prior to biofilter filtration.

\subsubsection{PCSWMM Entities}

The model entities consisted of one subcatchment, eight junctions, eight conduits, two ponds and an outfall. The cross-sections for the conduits were obtained from a full site survey and were assigned using the transect editor. A junction was placed at the appropriate locations where each of the crosssections was measured. The selected subcatchment parameters, based on literature for similar subcatchment characteristics, can be found in Table 9.1.

Table 9.1 Original subcatchment parameters.

\begin{tabular}{lc}
\hline \multicolumn{1}{c}{ Parameter } & Value \\
\hline Area (ha) & 0.85 \\
Width (m) & 10 \\
Flow length (m) & 850 \\
Slope (\%) & 4 \\
Imperv (\%) & 20 \\
N Imperv & 0.01 \\
Dstore Imperv. (mm) & 1.27 \\
Dstore Perv. (mm) & 2.54 \\
Zero Imperv. & 25 \\
Suction Head (mm) & 210 \\
Conductivity & 1.02 \\
Initial Deficit (fraction) & 0.187 \\
\hline
\end{tabular}




\subsubsection{SRTC Model Calibration}

In order to calibrate the model to an observed time series two events were selected: July 22 and July 30, 2008. These two events were selected because they were both large events and available on a continuous time series that was downloaded by on site flow loggers (see Table 9.2).

Table 9.2 Selected events for SRTC calibration.

\begin{tabular}{rcccc}
\hline Date & $\begin{array}{c}\text { Inflow volume } \\
\left(\mathrm{m}^{3}\right)\end{array}$ & $\begin{array}{c}\text { Precipitation } \\
(\mathrm{mm})\end{array}$ & $\begin{array}{c}\text { Rainfall } \\
\text { volume }\left(\mathrm{m}^{3}\right)\end{array}$ & $\begin{array}{c}\text { Runoff coeffi- } \\
\text { cient (fraction) }\end{array}$ \\
\hline 22-July-08 & 318 & 48.9 & 416 & 0.78 \\
30-July-08 & 261 & 43.2 & 367 & 0.61 \\
\hline
\end{tabular}

It recommended that more events be used for a calibration. However, for the sake of demonstrating the SRTC tool only two events will be used. These events were selected as they are both large events $(>40 \mathrm{~mm})$. In this model application it is hoped that the peak flow rates of the events are achieved rather than the lower intensity areas of the hydrographs.

The calibration was performed using the SRTC tool in PCSWMM 2010. This tool allows the user to select the parameters in SWMM entity layers (subcatchments, junctions, aquifers) with an allowable tolerance to be initially defined. Once the tolerance is defined an SRTC tool window allows the user to plot the observed and the computed time series, as well as to display maximum, mean and total rainfall plots with error display. Radio sliders located at the bottom of the window allow the user to change the parameter value within the pre-defined range in order to better match the time series plots (see Figure 9.1). From there the SRTC tool can create a scenario model with the new parameter values based on the slider bar positions. The calibrated subcatchent pararmeters are shown in Table 9.3.

Table 9.3 Calibrated subcatchment parameters.

\begin{tabular}{lc}
\hline \multicolumn{1}{c}{ Parameter } & Value \\
\hline Area (ha) & 0.85 \\
Width (m) & 160 \\
Flow length (m) & 850 \\
Slope (\%) & 4 \\
Imperv (\%) & 34 \\
N Imperv & 0.3 \\
Dstore Imperv. (mm) & 1.27 \\
Dstore Perv. (mm) & 2.54 \\
Zero Imperv. & 25 \\
Suction Head (mm) & 210 \\
Conductivity & 1.02 \\
Initial Deficit (fraction) & 0.187 \\
\hline
\end{tabular}


Using the PCSWMM 2010 SRTC Tool to Design a Compost Biofilter ...161

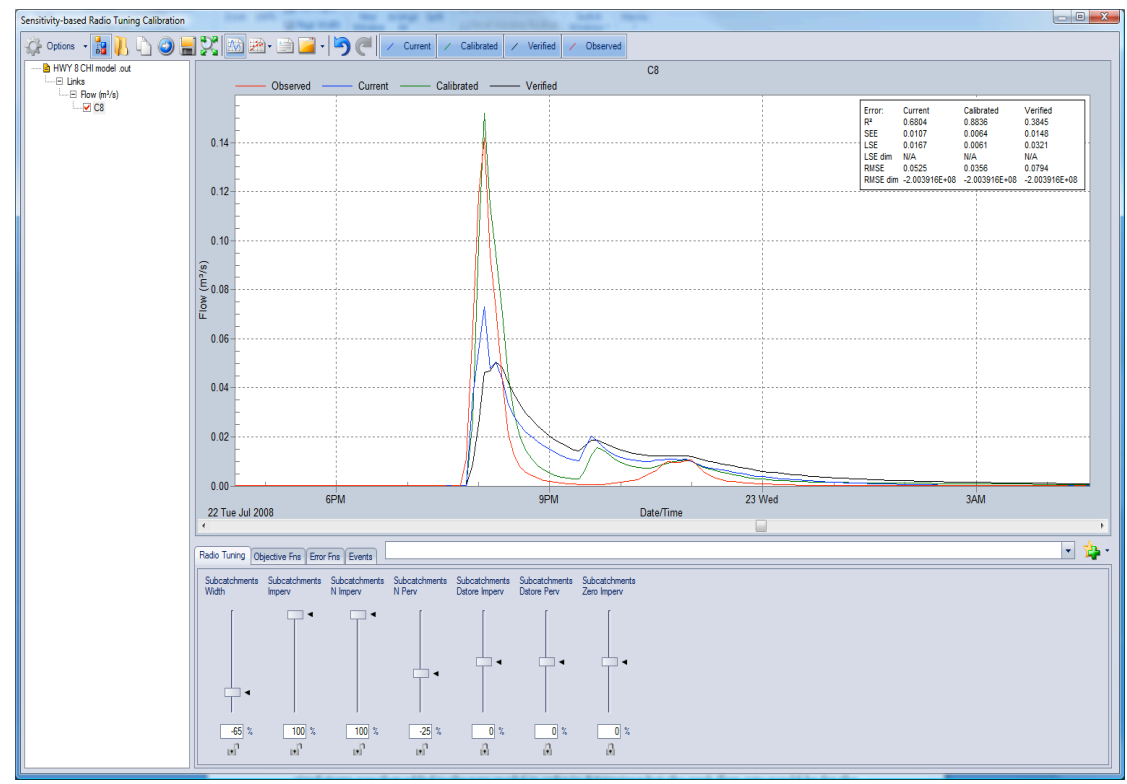

Figure 9.1 SRTC tool showing calibration of July 22, 2008.

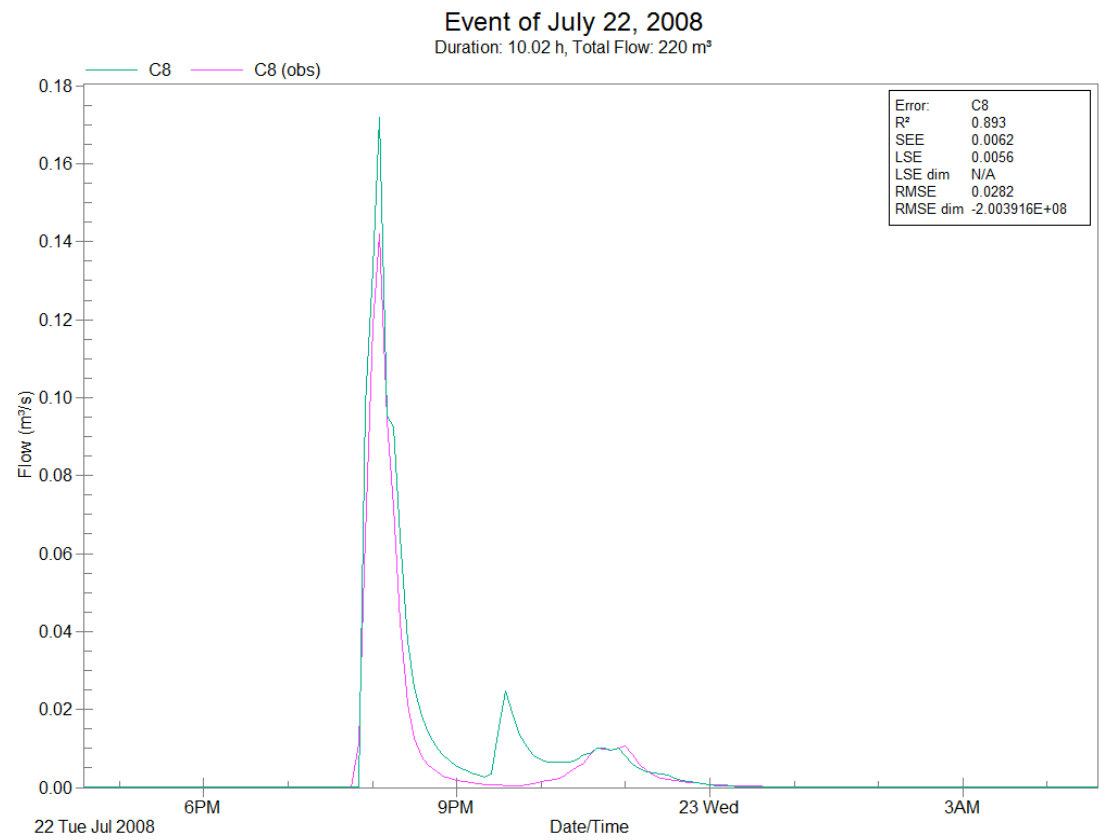

Figure 9.2 Calibrated hydrograph for July 22, 2008, event. 
Once the model was calibrated the new scenario was created with the representative site parameters. A design sized storm was then added to the calibrated model in order to determine what the peak flow rate would be for the site. Using this site data the desired compost biofilter dimensions can be determined. Figures 9.2 (above) and 9.3 present the calibrated hydrographs for the July 22nd and the July 30th, 2008, events.

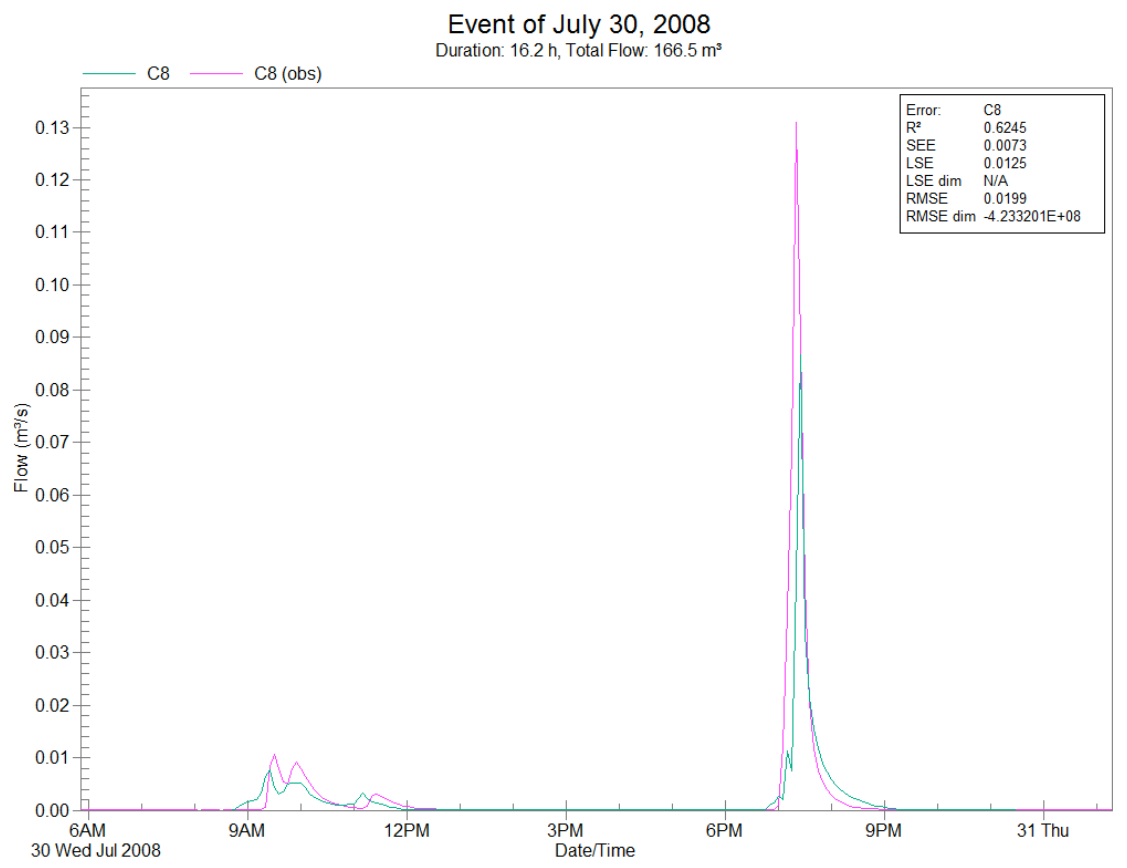

Figure 9.3 Calibrated hydrograph for July 302008 event.

\subsubsection{Design Storm}

In order to determine what the peak flow rate in a design sized storm would be for the Highway 8 remediation site, a design storm was added as a second rain gauge in PCSWMM. The design storm selected to determine the design of the compost biofilter was a Soil Conservation Service (SCS) 24 h type 2 event with a total rainfall of $100 \mathrm{~mm}$ (see Figure 9.4).

When the newly calibrated PCSWMM 2010 model was run the maximum flow was reported as being $0.2526 \mathrm{~m}^{3} / \mathrm{s}$ or $253 \mathrm{~L} / \mathrm{s}$. 


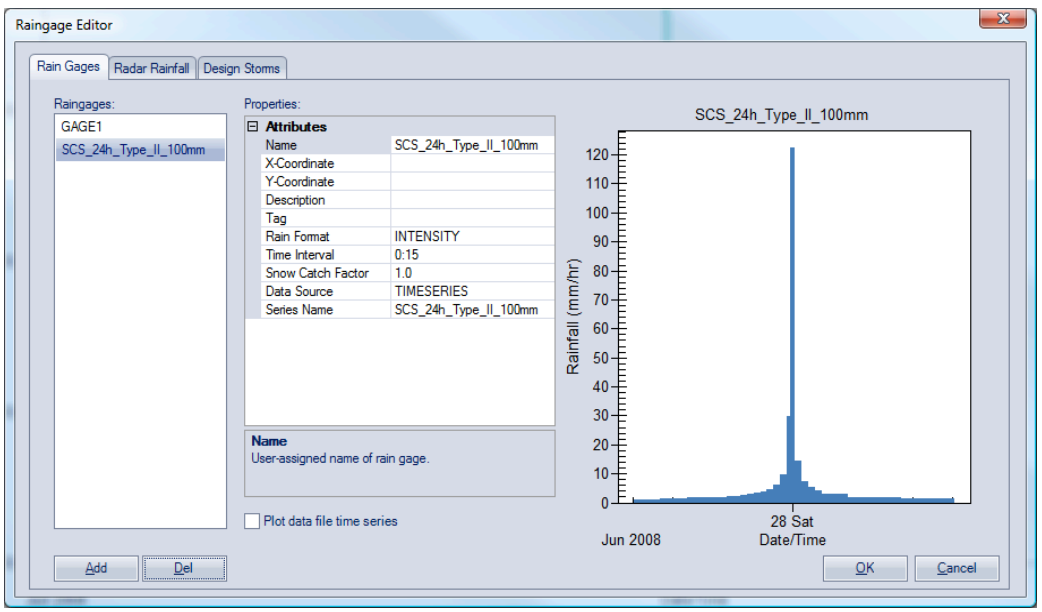

Figure 9.4 Design sized $100 \mathrm{~mm}$ event.

\subsection{Flow-through Capacity of a Compost Biofilter}

The flow-through capacity of the biofilter was calculated by plotting the relationship between the upstream biofilter water level and the biofilter flowthrough rate from seven observed events.

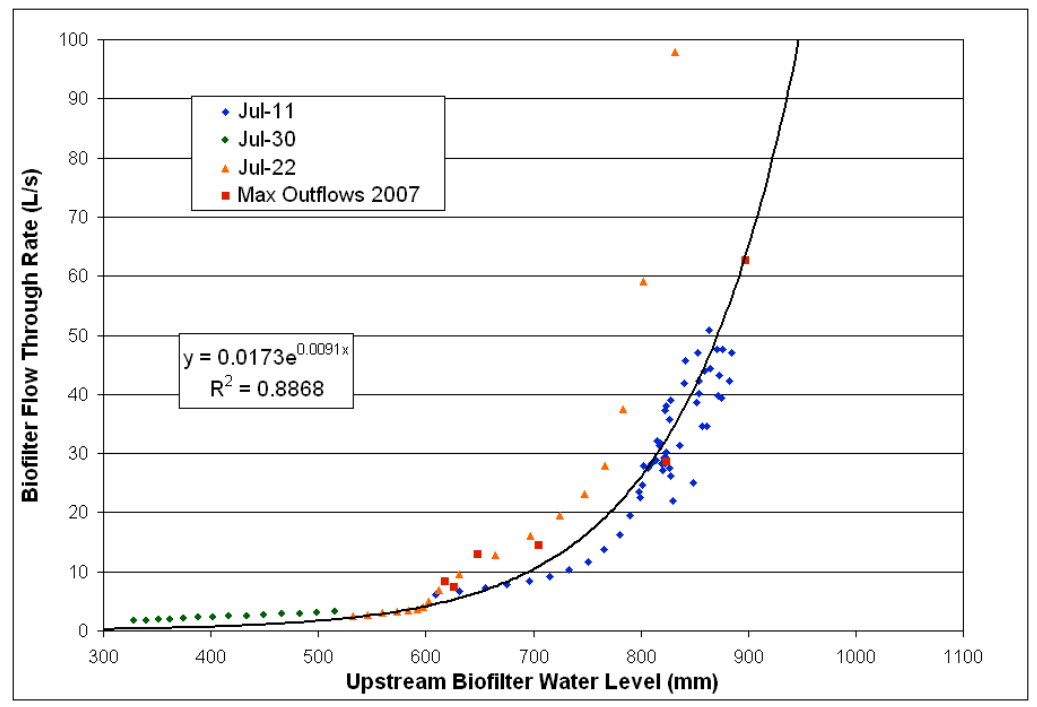

Figure 9.5 Biofilter flow-through rating curve. 
From this graph the flow-through capacity for the installed biofilter at the Highway 8 site was estimated to be $90 \mathrm{~L} / \mathrm{s}$. Thus the biofilter flow-though capacity (i.e. without overtopping) per unit cross-sectional area perpendicular to the flow direction for the compost material tested was $10 \mathrm{~L} / \mathrm{s} / \mathrm{m}^{2}$.

\subsection{Design of the Compost Biofilter}

Using the equation from Figure 9.5 the height of the biofilter can be calculated using the site dimensions and the peak flow rate using a biofilter material flow-through capacity of $10 \mathrm{~L} / \mathrm{s} / \mathrm{m}^{2}$.

$$
H=\frac{\ln \left(Q_{\max } / 0.0173\right)}{0.0091}
$$

where:

$$
\begin{aligned}
H & =\text { height of the biofilter }(\mathrm{m}), \text { and } \\
Q_{\max } & =\text { peak design flow rate }\left(\mathrm{L} / \mathrm{s} / \mathrm{m}^{2}\right) .
\end{aligned}
$$

To determine the cross-sectional area of the biofilter to install on the Highway 8 site the peak flow rate obtained from the PCSWMM model results was used to calculate the biofilter height. The SCS 24 h type 2 event with a total of $100 \mathrm{~mm}$ produced a peak flow rate of $253 \mathrm{~L} / \mathrm{s}$. The crosssectional transect of the ditch where the compost biofiler was to be installed had a bottom width of $4 \mathrm{~m}$ and a slide slope of $2: 1$. Using this data the height of the compost biofilter was then calculated using Equation 9.1 with the value $Q_{\max }=253$ :

$$
H=\frac{\ln (253 / 0.0173)}{0.0091}
$$

The height $H$ of the biofilter without overtopping was found to be $1053 \mathrm{~mm}$, approximately $1 \mathrm{~m}$. This means that the maximum height of water against the biofilter would be $1 \mathrm{~m}$. A safety factor of 2 was used, making the required height of the biofilter $2 \mathrm{~m}$.

Using the calculated value of the biofilter, the trapezoidal cross-sectional area $A_{\text {trapezoid }}$ was then determined by Equation 9.2, where $b_{1}$ and $b_{2}$ are the widths of the parallel sides of the trapezium and $H$ the distance between them:

$$
A_{\text {trapezoid }}=\left(\frac{\left(b_{1}+b_{2}\right)}{2}\right) \cdot H
$$

Using the values $b_{1}=4, b_{2}=6$ and $H=2$ in Equation 9.2 gives the result $A_{\text {trapezoid }}=10$. 
The cross-sectional area of the biofilter with a $4 \mathrm{~m}$ base width, a 2:1 slope and $2 \mathrm{~m}$ height would have a top width $12 \mathrm{~m}$ with a cross-sectional area of $32 \mathrm{~m}^{2}$. In order to check that the dimensions of the biofilter are accurate the seepage velocity $V_{\text {seepage }}$ can be calculated using Equation 9.3.

$$
V_{\text {seepage }}=\frac{Q_{\max }}{A_{\text {trapezoid }}} \cdot \frac{1}{1000}
$$

Using the values $Q_{\max }=253$ and $A_{\text {trapezoid }}=32$ in Equation 9.3 gives the result $V_{\text {seepage }}=0.008$.

Because the seepage velocity value is close to 0.01 it can be confirmed that the cross-sectional area for the compost biofilter is appropriate to prevent overtopping in a SCS $24 \mathrm{~h}$ type 2 event with a total rainfall of $100 \mathrm{~mm}$.

\subsection{Conclusion}

Compost biofilters have been found to be effective in removing pollutants from highway stormwater runoff. Use of the compost biofilter in place of a check dam also has the potential to allow for increased infiltration via ponding. However, it is important to ensure that the compost biofilter is designed properly using a suitable hydraulic model such as PCSWMM 2010 in order to avoid overtopping. The use of PCSWMM 2010 allows the designer to use the SRTC tool to easily calibrate the model with some assumed subcatchment parameters in order to better represent the existing site conditions. This study demonstrates PCSWMM model development and application for the design of the compost biofilter for the highway runoff treatment.

\section{References}

Finney K., Gharabaghi B., McBean E., Rudra R. and MacMillan G. 2010. Compost biofilters for highway stormwater runoff treatment. Water Quality Research Journal of Canada. In Press.

Makepeace, D. K., Smith, D. W. and Stanley, S. J. 1995. Urban stormwater quality: Summary of contaminant data. Critical Reiew. in Envir. Sci. and Technol., 25(2):93139.

Marsalek, D.K. 1995. Pollutant loads in urban stormwater: Review of methods for planning-level estimates. Journal of the American Water Resource Association, 27(2): 283-291.

Pitt, R. 2003. Sources of Stormwater Pollutants, Including Pollutant Buildup and Washoff. Extracted from Stormwater Quality Management. CRC Press, New York.

Taleban, V., Finney, K., Gharabaghi, B., McBean, E., Rudra, R. and Van Seters, T. 2009. Effectiveness of compost biofilters in removal of sediments from construction site runoff. Water Quality Research Journal of Canada, 44(1):71-80. 
